\title{
Effects of Rauwolfia serpentina L. Benth. ex Kurz (Serpentina) and
Costus igneus Nak. (Insulin plant) leaves crude extracts on the blood glucose levels of
alloxan induced albino rats
Gladys Fauni-Guirre ${ }^{1,2, *}$, Johnny A. Ching ${ }^{2}$
${ }^{1}$ Gen. Juan Castañeda Senior High School, Anabu II-A, City of Imus, Cavite, Philippines
Received - November 22, 2021; Revision - January 28, 2022; Accepted - February 27, 2022
Available Online - February 28, 2022
DOI: http://dx.doi.org/10.18006/2022.10(1).83.89 \\ KEYWORDS \\ Diabetes \\ Hypoglycemia \\ Phytochemicals \\ Insulin \\ Dietary supplement \\ Oxidative stress

\begin{abstract}
The prevalence of treatment failures from dietary patterns and oral medications associated with diabetes have generated adverse effects and are oftentimes expensive. Recently, food-based therapies such as Rauwolfia serpentina (serpentina) and Costus igneus (insulin plant) have been received much attention due to the urge for an alternative and safe solution against diabetes. Thus, the hypoglycemic effects of serpentina and insulin plant leaf crude extracts were determined on the blood glucose level of test rats. Twenty-four alloxan-induced male albino rats were subjected to this experimental study distributed into six groups in a completely randomized design. The negative control (NEG) comprised of diabetic rats receiving no treatment; while the positive control (MET) comprised of diabetic rats treated with metformin; experimental groups include IN1X and IN2X for the diabetic rats treated with extracts of insulin plant leaves administered once and twice daily and SER1X and SER2X for the diabetic rats treated with extracts of serpentina leaves administered once and twice daily. Results of the study revealed that both serpentina and insulin plant leaves crude extract demonstrated hypoglycemic effects due to the presence of zinc that potentiated insulin action. Further, the insulin plant improved glucose and insulin levels due to quercetin which reduced oxidative stress and protects DNA damage, $\beta$-amyrin and $\beta$-L-arabinose methyl glucoside which builds-up insulin for glucose metabolism. The presence of significant phytochemical contents in the insulin plant has been attributed to the stimulation of $\beta$ cells. In conclusion, insulin plant leaf crude extract elucidated better hypoglycemic activity than the serpentina plant leaf crude extract in the blood glucose levels of alloxan-induced diabetic rats.
\end{abstract}

* Corresponding author

E-mail: sydalg_637@yahoo.com (Gladys Fauni-Guirre)

Peer review under responsibility of Journal of Experimental Biology and Agricultural Sciences.

Production and Hosting by Horizon Publisher India [HPI] (http://www.horizonpublisherindia.in/).

All rights reserved.
All the articles published by Journal of Experimental Biology and Agricultural Sciences are licensed under a Creative Commons Attribution-NonCommercial 4.0 International License Based on a work at www.jebas.org.

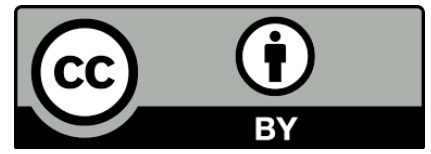




\section{Introduction}

The body's response to blood sugar is coordinated by a variety of mechanisms. To avoid the build-up of glucose in the blood, the insulin regulation, secretion, uptake, or breakdown of insulin in the body must not fail (Mahmoud et al. 2017). Drugs like alloxan monohydrate are used for introducing diabetes in animals (Qinna and Badwan 2015). According to Rohilla and Ali (2012), alloxan selectively destroys the insulin-producing $\beta$ cells found in the pancreas and causes diabetes. Alloxan monohydrate has been widely used to induce experimental diabetes due to its pancreaticselective $\beta$-cell toxin that induces random, rapid, and irreversible severe necrosis of $\beta$-cells and damages the pancreas (Ramadan et al. 2017). The insulin-producing cells in the pancreas are selectively destroyed and appeared to build a redox cycle with the development of superoxide radicals (Ojo et al. 2016). With a consequent lack of insulin secretion caused by alloxan induction, it has been widely used to induce experimental diabetes mellitus and many studies have been performed using this model to performed pancreatic damage (Helal et al. 2013).

There are several approaches currently available to treat diabetes including insulin therapy and treatment with biguanides such as metformin (Cheng and Fantus 2005). In recent years, several major insulin-sensitizing agents have been developed, including metformin as a modern oral hypoglycemic agent from the derivatives of a natural plant product (Lee et al. 2006). The frontline anti-diabetic drug metformin also known as dimethyl biguanide was developed from a plant-based molecule of Galega officinalis (Annadurai et al. 2012). Metformin causes weight reduction, improved insulin sensitivity, and lipid-lowering in both human and animal models of insulin resistance cell (Saenz et al. 2005) which is thought to have beneficial effects by activating the stress-activated kinase protein that signifies cellular stress in the treatment of type 2 diabetes and obesity (Leverge et al. 2003). Unfortunately, biguanide therapies have restricted viability with several side effects including hypoglycemia and weight gain. Therefore, the quest for alternative drugs from medicinal plants, such as $R$. serpentina (serpentina) and $C$. igneus (insulin plant), is fundamental to overcoming these problems (Kazeem et al. 2015).

Serpentina is globally recognized as antihypertensive phytomedicine and is a medicinally significant plant utilized to obtain drugs. It is said to appear in Sanskrit as an Ayurvedic medicine named Sarpgandha and Chandra which are referring to an antidote for snake-bite (Malviya and Sason 2016). Serpentina is a small annual shrub belonging to the family Apocynaceae that grows erect to a height of $30-110 \mathrm{~cm}$ in moist and shady places with a dark green slender stem, lance-shaped leaves with hairless blades measuring up to $2 \mathrm{~cm}$ long by $2.5 \mathrm{~cm}$ wide (Kumar et al. 2012). Known for its diversified ethnomedicinal usefulness, serpentina can cure snake bites, gastrointestinal tract disorders, breast cancer, and skin problems (Azmi et al. 2015). Many present studies emphasize the potential of serpentina as an anti-fungal, anti-inflammatory, anti-oxidant, anti-proliferative, anti-cancerous, anti-diuretic, anti-dysentery, and tranquilizing agent (Malviya and Sason 2016). The dynamic constituent of the serpentina is utilized adequately as commercial drugs in modern science (Azmi and Quereshi 2016).

On the other hand, the insulin plant has gained increased popularity in recent years due to its incredible cure for diabetes and contains a very potent effect of anti-diabetes (Aruna et al. 2014). Insulin plant, previously known as Costus pictus, is commonly referred to as fiery costus, spiral flag, spiral ginger, step ladder, or insulin plant. It is a perennial flowering plant belonging to the family of Costaceae and was recently separated from the family of Zingiberaceae due to the presence of spirally arranged leaves and rhizomes and no content of essential oils (George et al. 2007; Joshi et al. 2013). Due to its anti-hyperglycemic activity, the leaves of the insulin plant were utilized generally as a dietary food supplement in the management of diabetes (Gireesh et al. 2009). The leaves have been accounted to build insulin pools in the blood and assist with working up insulin in the body (Annadurai et al. 2012). Many diabetics claimed a lowering blood glucose levels after consuming the leaves of the insulin plant which lead to it being named insulin (Shetty et al. 2010). Although the insulin plant is being generally utilized for diabetes, there are still no available details about its formulation (Aruna et al. 2014) and possible business opportunities (Annadurai et al. 2012). Thus, this study was conducted to identify the effects of serpentina and insulin plant leaves crude extracts on the blood glucose levels of alloxaninduced albino rats.

\section{Materials and Methods}

\subsection{Research Design}

This is an experimental study carried out on 24 test rats that were subjected to the induction of alloxan monohydrate to increase blood glucose levels. The animals were distributed equally in a complete randomized design (CRD) employed with 6 groups namely, NEG (negative control) composed of diabetic rats receiving no treatment, MET (metformin drug) as a positive control composed of diabetic rats treated with commercial antidiabetic drug metformin, the animals of group IN1X and IN2X are diabetic rats treated with the extracts of insulin leaves administered once daily and twice daily, respectively, while the members of group SER1X and SER2X are diabetic rats treated with the extracts of serpentina leaves administered once and twice daily. Each group has four rats including replicates. Ethics review certification was issued by the DLSU-D Ethics Review Committee (DERC) before the conduct of the study. 


\subsection{Acclimatization and Maintenance of Laboratory Rats}

Control and experimental rats were raised as per the protocols of the Philippine Association of Laboratory Animal Science (PALAS) Code of Practice for the Care and "Use of Laboratory Animals" in the Philippines and John Hopkins University "Use of Experimental Animals (2002)". Rats were individually caged and kept in good condition in a well-ventilated and well-lighted room at room temperature in a veterinary clinic. Fifty grams of food pellets were fed to rats 3 times daily at $6 \mathrm{am}, 12 \mathrm{pm}, 6 \mathrm{pm}$, and water was provided ad libitum.

\subsection{Preparation and Administration of Drug Inducer}

After the acclimatization period, alloxan monohydrate was used to induce diabetes in rats through a single intra-peritoneal injection of a freshly prepared solution in normal saline at a dose of $150 \mathrm{mg} / \mathrm{kg}$ body weight once a day for three successive days (Azmi et al. 2015).

\subsection{Preparation of Different Treatments}

Standardized Metformin Gludin ${ }^{\circledR}$ oral hypoglycemic tablets were prepared based on the weight of rats using the standard dose of 500 $\mathrm{mg} / \mathrm{kg}$ and further diluted in distilled water. Fresh leaves of the test plants about $2 \mathrm{~kg}$ were collected from a local horticultural farm. The leaves were collected, cleaned, and washed with distilled water, shaded dried for 21 days, and coarsely powdered; this powder was well soaked in a plastic container at about 8L 95\% laboratory-grade ethyl alcohol. It was kept overnight at room temperature. The supernatant was collected and evaporated to dryness using a rotary evaporator (Heidolph $\left.{ }^{\circledR}\right)$ and the final residue was lyophilized using a lyophilizer (Azmi et al. 2015; Ashwini et al. 2015; Nicolas et al. 2016).

\subsection{Administration of Treatment}

Prepared oral hypoglycemic tablets and leaf crude extract treatments were administered to diabetic rats via the oral route using the gavage method. A standard dosage of $500 \mathrm{mg} / \mathrm{kg}$ body weight for the Metformin and $250 \mathrm{mg} / \mathrm{kg}$ body weight for the leaf crude extracts were given at every $24-\mathrm{hr}$ interval during the entire period of the experiment. The treatments were inserted into the mouth of the albino rats using the flexible ball tip of a needle. The rats were restrained by holding onto the scuff or the loose folds of the skin on the neck and back portion of the rat. Once the rat was properly restrained, the gavage needle was inserted carefully into the mouth of the rat to ensure proper administration of treatment. The administration started 72-hours post-induction of alloxan monohydrate at an interval of every 24 hours thereafter for 21 days (Gireesh et al. 2009; Shetty et al. 2010; Nicolas et al. 2016).

\subsection{Data Collection and Statistical Analysis}

Blood samples were collected 3 hours after the administration of the treatments. A drop of blood was placed on the blood glucose test strips and the strip was inserted on a glucose meter. The reading displayed on the screen was recorded. The results were expressed in terms of a milligram per decilitre of blood. Rats with fasting blood glucose of more than $200 \mathrm{mg} / \mathrm{dl}$ were considered diabetic and included in the experimentation after 72-hour postinduction of alloxan monohydrate. Results of blood glucose levels were compared with the standard hematologic data for rats to investigate the efficacy of serpentina and insulin plant leaves crude extract against diabetes mellitus (Gireesh et al. 2009).

Two-way analysis of variance (ANOVA) was used to compare for significant differences between the treatments with serpentina and insulin plant leaves crude extract. Likewise, a posthoc test was used to find out if a significant interaction among the groups in the hematologic data. All statistical analyses were done at a 5\% probability level.

\section{Results}

\subsection{Blood Glucose Test Analysis}

Blood glucose tests performed in positive control and treated rats showed remarkable results (Figure 1). The blood glucose level of all treatment groups showed a significant increase at post-induction of alloxan monohydrate. Administration of alloxan monohydrate led to a highly significant increase in the blood glucose level of the rats when compared to the pre-induction period.

One-week post-treatment, all rats that received different treatments showed a notable decrease (>23 mg/dl) of blood glucose level. The highest value of blood glucose decrease was observed from the IN2X group while the NEG group has the least decrease. After two weeks, the IN1X group continued to show a decreasing blood glucose level. On the other hand, IN2X and MET group started to increase their blood glucose level while SER2X is almost the same as the status of the NEG (within 222-263 mg/dl). However, all treatment groups had not reverted to normal blood glucose levels (>200 mg/dl). After three weeks, the MET group showed the highest decrease of blood glucose level while the lowest decrease was observed from the IN1X group. On the other hand, the constant blood glucose level was observed from the IN2X group. Comparing the decrease, SER1X and NEG group have a comparable increase of blood glucose level, while SER2X group already started to show the highest increase of blood glucose value.

It is worth noting that the decrease (or increase) during the $1^{\text {st }}$ week, $2^{\text {nd }}$ week, and $3^{\text {rd }}$ week were highly significantly varied $(\mathrm{p}<0.05)$ within treatment groups (Table 1). During the first week, IN2X, IN1X, and MET groups, statistically, have a 
comparable decrease while NEG has the least decrease among the treatments. Hence, while constant blood glucose level was observed in IN2X during the $2^{\text {nd }}$ and $3^{\text {rd }}$ week, it has statistically the highest decrease among the treatments for three weeks. Although NEG has the least decrease during the $1^{\text {st }}$ week, it has statistically the lowest increase during the $2^{\text {nd }}$ week. On the other hand, a significant decrease in IN1X was observed during the $2^{\text {nd }}$ week only, however, the MET group started to decrease significantly with the highest decrease observed among the treatments during the $3^{\text {rd }}$ week. Comparing the decrease in the blood glucose level of the different treatment groups during the $1^{\text {st }}$ week, $2^{\text {nd }}$ week, and $3^{\text {rd }}$ week, the IN2X group has the highest decrease at $221 \mathrm{mg} / \mathrm{dl}$ while SER2X has the lowest decrease at $225 \mathrm{mg} / \mathrm{dl}$.

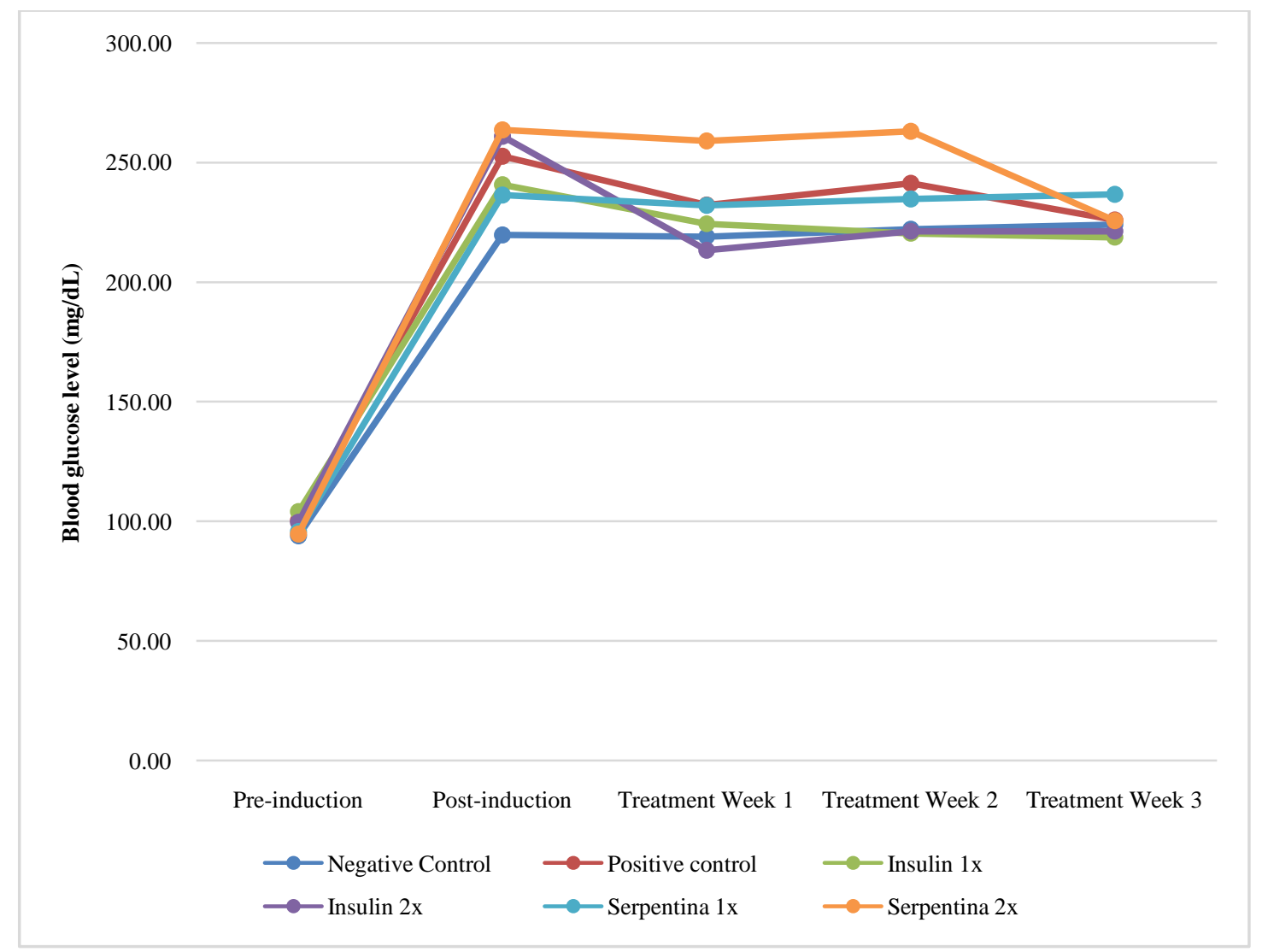

Figure 1 Average blood glucose level on rats prior to and after alloxan monohydrate induction and post treatment

Table 1 Average decrease (increase) of blood glucose value $(\mathrm{mg} / \mathrm{dl})$ on rats prior to and after alloxan monohydrate induction and 3 weeks after treatment

\begin{tabular}{|ccccc|}
\hline Treatment Group & Pre-induction & Post-induction & Post-treatment after 3 weeks & Difference \\
\hline NEG & $94.00 \pm 1.41^{\mathrm{A}}$ & $219.67 \pm 11.90^{\mathrm{B}}$ & $224.00 \pm 13.95^{\mathrm{B}}$ & $4.33 \pm 3.09^{\mathrm{X}}$ \\
\hline MET & $103.33 \pm 9.39^{\mathrm{A}}$ & $252.67 \pm 8.65^{\mathrm{B}}$ & $226.00 \pm 12.33^{\mathrm{C}}$ & $-26.67 \pm 4.50^{\mathrm{Y}}$ \\
\hline IN1X & $104.00 \pm 6.16^{\mathrm{A}}$ & $240.67 \pm 12.23^{\mathrm{B}}$ & $218.67 \pm 14.73^{\mathrm{C}}$ & $-22.00 \pm 3.56^{\mathrm{Y}}$ \\
\hline IN2X & $99.67 \pm 4.92^{\mathrm{A}}$ & $261.00 \pm 11.86^{\mathrm{B}}$ & $221.33 \pm 7.72^{\mathrm{C}}$ & $-39.67 \pm 8.50^{\mathrm{Z}}$ \\
\hline SER1X & $95.67 \pm 1.25^{\mathrm{A}}$ & $236.33 \pm 12.28^{\mathrm{B}}$ & $236.67 \pm 11.79^{\mathrm{B}}$ & $0.33 \pm 0.94^{\mathrm{X}}$ \\
\hline SER2X & $94.67 \pm 6.02^{\mathrm{A}}$ & $263.67 \pm 28.24^{\mathrm{B}}$ & $225.66 \pm 28.61^{\mathrm{C}}$ & $-18.92 \pm 0.69^{\mathrm{Y}}$ \\
\hline
\end{tabular}

*Normal values (Emordi et al.2016; Qinna and Badwan 2015; Helal et al. 2013); Different letters denote statistically significant ( $\mathrm{p}<0.05)$. Letters $\mathrm{ABC}$ compare the blood glucose levels during pre- and post-induction of alloxan and after 3-week treatment. Letters XYZ compare the difference (3-week treatment - post-induction) of blood glucose level between treatment groups; Legend: $\mathrm{NEG}=$ negative control, $\mathrm{MET}=$ metformin, IN1X = Insulin leaf crude extract administered once daily, IN2X = Insulin leaf crude extract administered twice daily, SER1X = Serpentina leaf crude extract administered once daily, SER $2 X=$ Serpentina leaf crude extract administered twice daily.

Journal of Experimental Biology and Agricultural Sciences http://www.jebas.org 


\section{Discussion}

Insulin plant leaf crude extract has shown a potent anti-diabetic effect and ameliorates hyperglycemia which enhanced insulin secretion (Gireesh et al. 2009; Joshi et al. 2013; Ashwini et al 2015). The administration of insulin plant leaf extract on rats showed the highest marked decrease in the blood glucose level measured in $\mathrm{mg} / \mathrm{dl}$ in the serum one week post-treatment than the administration of serpentina plant leaf extract in double dosage. These findings can be attributed to the strong $\alpha$-amylase inhibitory activity of the insulin plant, which was not observed in the properties of serpentina. The enzyme $\alpha$-amylase hydrolyzes the $\alpha$ bonds of large $\alpha$-linked polysaccharides such as glycogen and starch to yield glucose and maltose. In addition, $\alpha$-amylase inhibitors bind to $\alpha$-bond of polysaccharide and prevent the breakdown of polysaccharide into monosaccharide and disaccharide, thus preventing the absorption of glucose in the bloodstream (Aruna et al. 2014). Therefore, even in a single dose, the insulin leaf crude extract showed improvement in the blood glucose level of rats than the administration of serpentina leaf crude extract twice a day. Moreover, based on the results of phytochemical analysis, insulin plant crude leaf extract contains highly significant quantities of polyphenolic compounds, such as flavonoids, than serpentina crude leaf extract. Consumption of foods high in polyphenolic compounds was associated with a lower risk of diabetes (Hanhineva et al. 2010). Further, recent studies have demonstrated that insulin plant leaf crude extract was effective in preventing insulin resistance by improving insulin sensitivity at the peripheral level through its anti-oxidant and inflammatory effects that involve stress-sensitive signaling cascade at the molecular level (Ashwini et al. 2015). The lowest decrease of blood glucose level was observed in IN1X three weeks posttreatment but proved to be effective in maintaining the constant value of blood glucose level and body weight as shown in the IN2X group. Hence, it is interesting to note that the prolonged use of the insulin leaf crude extract may potentiate the hypoglycemic action due to the release of insulin-sensitizing action against oxidative stress and regeneration of the $\beta$ cells in a slow action after three weeks. This agreed with the findings of Emordi et al. (2016) that show a dramatic decrease in plasma glucose concentration as a result of the slow recovery of insulin releases from the regenerated $\beta$ cells of the damaged pancreas. The blood glucose lowering effect of insulin plant crude leaf extract was due to its role as an antioxidant enzyme that protects against free radicals that contribute in the oxidative stress by scavenging oxygen free radicals, caused by the pancreatic $\beta$ cell necrosis which released abundant free radicals (Hoque et al. 2011). One of the noteworthy aspects of the insulin plant leaf crude extract was the anti-inflammatory actions of phenolic compounds that are hence the possible explanation for the improvement in insulin sensitivity and glycemic control seen in the study of Dragan et al.
(2015). This conformed to the study of Pitchai et al. (2010) that the productive effects were majorly due to the presence of various secondary metabolites which enhance the versatile ethnopharmacological properties of medicinal plants with almost negligible side effects. Therefore, this study led to the idea that insulin plant leaf crude extract showed an anti-hyperglycemic effect and improvement in insulin sensitivity in the alloxaninduced diabetic rats with no side effects exhibited.

It was worth noting that the effect of serpentina leaf crude extract in lowering the blood glucose level was slower as compared to insulin plant leaf crude extract, primarily due to its mode of action in stimulating the release of insulin from the pancreatic $\beta$ cells through ATP-sensitive potassium channels (Akbar 2011). Both short and long-term antidiabetic activities of serpentina have been reported by Azmi and Quereshi (2016). Advanced computational studies on alkaloids of serpentina highlighted the role of insulin receptor activators and aldose reductase inhibitors, which strengthens the anti-diabetic activity of this plant (Pathania et al. 2013). In this study, the administration of serpentina on diabetic rats caused by an alloxan-induced drug proved effective as expressed by the decreased level of blood glucose one week posttreatment. The hypotriglyceridemic effect of serpentina was due to its ability to enhance insulin sensitivity for the receptor due to a significant amount of alkaloids and polyphenolic compounds which are involved in improving glucose and lipid homeostasis (Azmi et al. 2015). Therefore, serpentina can be effective in improving insulin resistance which can improve glucose uptake in target tissues and stimulate anabolic processes of insulin-like glycogenesis and lipogenesis. Further, the hypoglycemic effect of serpentina was also due to its extra-pancreatic action via inhibiting fructose absorption in the intestine and reducing insulin resistance. However, it was not observed in this study, since based on the result, constant supplementation of serpentina for two weeks posttreatment recorded the lowest decrease of blood glucose. In the same manner, a similar result was reported by Nicolas et al. (2016) that the effect of serpentina was slower in decreasing the blood glucose level.

\section{Conclusion}

Based on the result of this study, the antioxidant activities of the insulin plant might have elucidated better compared to that of serpentina since the insulin plant caused a more consistent decreasing pattern of blood glucose level which was also comparable to metformin drug. The insulin plant exerts an antidiabetic effect by stimulating insulin secretion from the pancreas under insulin-resistant conditions. Insulin plant leaf extract decreases hyperinsulinemia by improving insulin sensitivity through its anti-oxidant and anti-inflammatory effects caused by $\beta$ amyrin. At a molecular level, the insulin plant decreased activation 
of molecules involved in stress-sensitive signaling cascade and resulted in the downregulation of pro-inflammatory cytokines. In addition, the presence of quercetin, a class of flavonoids, in the insulin plant, plays a vital role in the reduction of oxidative stress, low-grade inflammation, and down-regulates the gene expression and production of the pro-inflammatory cytokines in the blood cells. The high content of phenolic compounds and flavonoids in the insulin plant was postulated to contribute to its antidiabetic activity through scavenging oxygen free radicals. Hence, the blood glucose lowering effect of insulin plant leaf crude extract was due to its role as an antioxidant enzyme that protects against free radicals that contribute to oxidative stress which leads to the induction of systemic insulin resistance cells.

Furthermore, serpentina obtained the lowest reduction in the blood glucose level in the entire period of three-week post-treatment. The gradual reduction of blood glucose level by serpentina was due to its mode of action that stimulates the release of insulin from pancreatic $\beta$-cells through ATP-sensitive potassium channels. Moreover, the anti-diabetic activity of serpentina is due to the increase in glucose utilization by delaying or preventing glucose absorption that is caused by the potential inhibitors of alphaglucosidase and alpha-amylase that have been yielded in the plant. But since, in this case, alloxan-induction caused permanent diabetes due to the destruction of the $\beta$ cells and therefore, the available pancreatic $\beta$ cells to be stimulated were not present or if present, in insignificant number.

\section{Conflict of Interest}

The authors would hereby like to declare that there is no conflict of interests that could arise.

\section{Acknowledgments}

The authors express their gratitude to Dr. Mary Jane Flores, Dr. Airill Mercurio, Dr. Joseph Samonte, Dr. Marcelino Siladan, and Dr. Amparo Sulam of the College of Science and Computer Studies Graduate Studies of De La Salle University-Dasmariñas for their intelligent inputs for the completion of this study.

\section{References}

Akbar, S. (2011). Andrographis paniculata: A review of pharmacological activities and clinical effects. Alternative Medical Review, 16 (1), 66-77.

Annadurai, R., Jayakumar, V., Mugasimangalam, R., \& Katta, M. (2012). Next generation sequencing and de novo transcriptome analysis of Costus pictus D. Don, anon-model plant with potent anti-diabetic properties. Biomed Central Genomics, 663(13), 2-13.
Aruna, A., Nandhini, R., Karthikeyan, V., \& Bose, P. (2014). Synthesis and characterization of silver nanoparticles of insulin plant (Costus pictus D.Don) leaves. Asian Journal of Biomedical and Pharmaceutical Sciences, 4(34), 1-6.

Ashwini, S., Bobby, Z., Joseph, M., Jacob, S., \& Padmapriya, R. (2015). Insulin plant (Costus pictus) extract improves insulin sensitivity and ameliorates atherogenic dyslipidaemia in fructose induced insulin resistant rats: molecular mechanism. Journal of Functional Foods, 17, 749-760.

Azmi, M., \& Qureshi, S. (2016). Rauwolfia serpentina improves altered glucose and lipid homeostasis in fructose-induced type 2 diabetic mice. Pakistan Journal of Pharmaceutical Science, 29 (5), $1629-1624$

Azmi, M., Quereshi, S., Rais, S., \& Sultana, S. (2015). Methanolic root extract of Rauwolfia serpentina lowers atherogenicdyslipidemia, atherosclerosis and glysylation indices in type 1 diabetic mice. Journal of Applied Pharmaceutical Science, 5(8), 61-67.

Cheng, A., \& Fantus, I. (2005). Oral antihyperglycemic therapy for type 2 diabetes mellitus. Canadian Medicine Association Journal, $172,213-226$

Dragan, S., Andrica, F., Serbian, M., \& Timar, R. (2015). Polyphenols rich natural products for treatment of diabetes. Current Medicinal Chemistry, 22 (1), 214-221.

Emordi, J., Agbaje, E., Oreagba, I., \& Iribhogbe, O. (2016). Antidiabetic and hypolipidemic activities of hydroethanolic root extract of Uvaria chamae in streptozotocin-induced diabetic albino rats. Biomed Central Complementary and Alternative Medicine, 16(1), 468.

George, A., Thankamma, A., Devi, V., \& Fernandez, A. (2007). Phytochemical investigation of insulin plant (Costuspictus). Asian Journal of Chemistry, 19 (5): 3427-3430.

Gireesh, G., Thomas, S., Joseph, B., \& Paulose, C. (2009). Antihyperglycemic and insulin secretory activity of Costus pictus leaf extract in streptozotocin induced diabetic rats and in in-vitro pancreatic islet culture. Journal of Ethnopharmacology, 123, 470474.

Hanhineva, K., Torronen, R., Bondia-Pons, I., Pekkinen, J., et al. (2010) Impact of dietary polyphenols on carbohydrate metabolism. International Journal of Molecular Sciences, 11(4), 1365-1402.

Helal, E., Abd El-Wahab, S., Refaey, H., \& Mohammad, A.A. (2013). Antidiabetic and antihyperlipidemic effect of Balanites 
aegyptiaca seeds (aqueous extract) on diabetic rats. The Egyptian Journal of Hospital Medicine, 52, 725-739.

Hoque, N., Imam, Z.M., Akter, D., Mazumber, M., et al. (2011). Antioxidant and antihyperglycemic activities of methanolic extract of Glinus oppositifolius leaves. Journal of Applied Pharmacology Sciences, 1 (7), 50-53.

Joshi, B., Munot, H., Hardikar, M., \& Kulkarni, A. (2013). Orally active hypoglycemic protein from Costusigneus: An in vitro and in vivo study. Biochemical and Biophysical Research Communications, 436, 278-282.

Kazeem, M., Akanji, M., \& Yakubu, M. (2015). Amelioration of pancreatic and renal derangements in streptozotocin-induced diabetic rats by polyphenols extract of ginger (Zingiber officinale) rhizome. Pathophysiology, 22, 203-209.

Kumar, A., Dora, J., Sing, A., \& Tripathi, R. (2012). A review on king of bitter (kalmegh). International Journal of Research, Pharmacology and Chemistry, 2(1), 116-124.

Lee, Y., Kim, W., Kim, K., Yoon, M., et al. (2006)Berberine, a natural plant product, activates AMP-Activated protein kinase with beneficial metabolic effects in diabetic and insulin-resistant states. Diabetes, 55, 8.

Leverge, X.M., Guigas, B., Detaille, D., Batandier, C., et al. (2003) Mitochondrial metabolism and type 2 diabetes: a specific target of metformin. Diabetes metabolism, 29(6), 88-94.

Mahmoud, M., El-Ashry, F.E., El-Maraghy, N., \& Fahmy, A. (2017). Studies on the antidiabetic activities of Momordica charantia fruit juice in streptozotocin-induced diabetic rats. Pharmaceutical Biology, 55(1), 758-765.

Malviya, A., \& Sason, R. (2016). The phytochemical and pharmacological properties of Serpaganda: Rauwolfia serpentina. International Journal of Research in Ayush and Allied Systems, 3(1), 473-478.

Nicolas, K.M., Visaya, K., \& Cauinian, E. (2016). Blood glucose and cholesterol levels in alloxan-induced diabetic mice after oral administration of serpentine (Andrographis paniculata) and papait
(Mollugo oppositifolia) aqueous extracts. Philippine Journal of Veterinary and Animal Sciences, 42(2), 112-119

Ojo, A., Adanlawo, I., \& Ojo, O. (2016). Ameliorative potentials of saponins from Helianthus annus roots on hepatoprotective and some kidney function indices of alloxan-induced diabetic rats. Journal of Pharmacology, 3, 73-79.

Pathania, S., Randhawa, V., \& Bagler, G. (2013). Prospecting for novel plant-derived molecules of Rauwolfia serpentina as inhibitors of aldose reductase, apotent drug target for diabetes and its complications. PLOS ONE, 8(4), e61327. https://doi.org/10.1371/journal.pone.00613278: e61327.

Pitchai, D., Manikkam, R., Rajendran, S., \& Pitchai, G. (2010). Database on pharmacophore analysis of active principles from medicinal plants. Bioinformation, 5, 43-45.

Qinna, N.A., \& Badwan, A. (2015). Impact of streptozotocin on altering normal glucosehomeostasis during insulin testing in diabetic rats compared tonormoglycemic rats. Drug Design Development and Therapy, 9, 2515-2525.

Ramadan, B., Schaalan, M., \& Tolba, A. (2017). Hypoglycemic and pancreatic protective effects of Portulaca oleracea extract in all oxan-induced diabetic rats. BMC Complementary and Alternative Medicine, 17, 37.

Rohilla, R., \& Ali, S. (2012). Alloxan-induced diabetes: mechanism and effects. International Journal of Research in Pharmaceutical and Biomedical Science, 3, 819-820.

Saenz, A., Fernandez-Esteban, I., Matai, A., Ausejo, M., Roque, M., \& Moher, D. (2005). Metformin monotheraphy for type 2 diabetes mellitus. Cochrane database system review, 20, CD002966.

Shetty, A., Choudhury, D., Nair, V., Kuruvilla, M., \& Kotian, S. (2010). Effect of insulin plant (Costus igneus) leaves on dexamethasone-induced hyperglycemia. International Journal of Ayurveda Research, 1(2), 101-102.

Use of Experimental Animals (2002). Available at https://web.jhu.edu/animalcare/UpdatedBlueBookNoDrugFormula ry.pdf access on 29th April 2021. 